\title{
The Future Role, Clinical and Economic Implications of Cardiac Magnetic Resonance (CMR) Imaging in Non-Ischemic Dilated Cardiomyopathy
}

Malka Yahalom ${ }^{1}$ and Yoav Turgeman ${ }^{1,2 *}$

${ }^{1}$ Heart Institute, HaEmek Medical Center, Afula, Israel

${ }^{2}$ Rappaport School of Medicine, Technion, Haifa, Israel

\begin{abstract}
The use of cardiovascular magnetic resonance (CMR) imaging for assessing and evaluating cardiovascular pathologies is growing rapidly. This non-invasive, non-ionizing technology has multiple advantages compared with the X-ray or gamma ray derived technologies.

For instance, CMR is a non-invasive and sensitive tool for detecting islands of fibrosis. Cardiac fibrosis may occur in both ischemic and non-ischemic cardiac pathologies. This finding has a negative impact on sudden cardiac arrhythmic death.

A correlation has been found between CMR data and histo-pathologic findings. The presence of this pathology among normal looking fibers indicates the presence of non-functional areas that may evoke malignant arrhythmia, and contribute to deterioration in cardiac mechanical function.

By using this non-invasive modality, the role of cardiac fibrosis as a potential source for cardiac arrhythmia and or LV dysfunction can be better evaluated and managed. This finding may serve as a useful tool for risk stratification of patients with borderline indication for primary AICD implantation.
\end{abstract}

Keywords: Myocardial fibrosis; Automatic internal cardioverter defibrillator; Cardiovascular magnetic resonance imaging

Abbreviations: AICD: Automatic Internal Cardioverter Defibrillator; CMR: Cardiovascular Magnetic Resonance; NIDC: NonIschemic Dilated Cardiomyopathy; SCD: Sudden Cardiac Death; LGE: Late Gadolinium Enhancement

\section{Introduction}

As a result of rapid technological progress, including advancements in hardware, software, and the development of Late Gadolinium Enhancement Imaging (LGE) in the late 1990s, cardiovascular magnetic resonance Imaging (CMR) has been established as an important method in the diagnosis of cardiovascular disease, including ischemic (by identification of even small subendocardial infarcts) and Non-Ischemic Cardiomyopathies (NICM) [1].

Many clinical and experimental studies have demonstrated the superiority of CMR over imaging modalities such as nuclear medicine or transthoracic echocardiography [2]. It has been emphasized that the particular strengths of CMR are: the ability to overcome anatomical limitations such as a poor acoustic-windowand comprehensive answer to various aspects of cardiac diseases [3-8]. The absence of imaging radiation during the examinations, is especially important inpediatric and adult congenital heart disease, where repeated assessment is required [9].

It is accepted by most researchers that challenges for future development of the role of CMR in clinical routine will include not only technical improvement, but also optimized cost-efficiency and extended education and training [2]. There is an abundance of articles concerning the utility of CMR, which is well established in Europe and in North America [10], for patient risk stratification and its potential role in important management decisions, such as cardiac resynchronization therapy and defibrillation placement [11].

The relative prevalence of NIDC is 1:2500 among adult population. Patients withNICM at higher risk for sudden cardiac death (SCD) who will benefit from Internal Cardioverter Defibrillation (ICD), remains a major challenge in clinical cardiology [12]. This entity is also associated with significant morbidity and mortality (up to $20 \%$ in 5 years) due to progressive heart failure and SCD [13].

Due to the relatively common prevalence of the disease, the cost of ICD implantation, the inherent ICD Implant risks, and the risk (clinical and psychological) of inappropriate shocks [13], the current American College of Cardiology / American Heart Association Guidelines state that the ICD therapy is indicated for primary prevention in patients with NIDC with ejection fraction (LVEF) $\leq 35 \%$, who are in the NewYork Heart Association (NYHA) functional class II or III [14].

It should be noted that the rate of appropriate discharge in those patients is only $5.1 \%$ per year, which limits the use of this approach.It was also noted that most of the patients with NIDC who died from SCD had a normal ejection fraction $[15,16]$.

Cardiac fibrosis has a significant role in the mechanism of arrhythmia generation and heart failure pathophysiology. The edit value of cardiac fibrosis detectedby CMR in evaluating and managing this group of patients is discussed.

\section{Materials and Methods}

A literature search focusing on cardiac fibrosis detected by CMR

*Corresponding author: Yoav Turgeman, Heart Institute, HaEmek Medical Center Afula, Israel, Tel: 972-4-6494124; Fax: 972-4-6491414; E-mail: Yoav_t@clalit.org.il

Received August 04, 2014; Accepted October 15, 2014; Published October 17 2014

Citation: Yahalom M, Turgeman Y (2014) The Future Role, Clinical and Economic Implications of Cardiac Magnetic Resonance (CMR) Imaging in Non-Ischemic Dilated Cardiomyopathy. J Vasc Med Surg 2: 161. doi: 10.4172/2329-6925.1000161

Copyright: @ 2014 Yahalom M, et al. This is an open-access article distributed under the terms of the Creative Commons Attribution License, which permits unrestricted use, distribution, and reproduction in any medium, provided the original author and source are credited. 
Citation: Yahalom M, Turgeman Y (2014) The Future Role, Clinical and Economic Implications of Cardiac Magnetic Resonance (CMR) Imaging in Non-Ischemic Dilated Cardiomyopathy. J Vasc Med Surg 2: 161. doi: 10.4172/2329-6925.1000161

was undertaken in PubMed for the period of 2003-2014. The utility of this technique and its potential application in sudden cardiac death was screened and presented.

\section{Cardiac fibrosis}

In heart failure, fibrosis of the heart replace viable myocetescreating potential substrate for arrhythmiageneration. Gulati, et al. evaluated the idea that the presence of fibrosis (scar) within the left ventricle would add a prognostic accuracy to left ventricular ejection fraction in assessing the risk of SCD in patients with NIDC $[13,17]$.

A prospective longitudinal evaluation of 472 patients with NIDC was conducted by them in a single center in London, England, between the years 2000-2008. CMR imaging with Late GadoliniumEnhancement (LGE-CMR) was used to determine the presenceor absence of ventricular midwall fibrosis.

The presence of LGE correlated histologically with the presence of fibrosis, resembling previous data [18]. In this study, the presence, location and extent of fibrosis were assessed. A multivariate analysis found that the presence of midwall fibrosis was highly significant risk factor for the primary endpoint of total mortality, and to a lesser extent, the percentage of this phenomenon.

In another study, Neilan et al (from Boston) had shown similar data [18]. In a recent study from Padova, Italy, it was shown that LV-LGE is a powerful and independent prediction of malignant arrhythmicprognosis [19].

\section{Summary and Conclusions}

In summary, there is agreement among researchers about the potential utility of LGE-CMR for assisting the risk of SCD and subsequent ICD implants. All agreed that a single-center studies need confirmation by large multi-center trials, before generalized use of this new modality can be recommended.

\section{References}

1. Karamitsos TD, Neubauer S (2013) Detecting diffuse myocardial fibrosis with CMR: the future has only just begun. JACC Cardiovasc Imaging 6: 684-686.

2. Pilz G, Heer T, Harrer E, Ali E, Hoefling B (2009) Clinical applications of cardiac magnetic resonance imaging. Minerva Cardioangiol 57: 299-313.

3. Pennell DJ, Schtem UP, Higgins CB, Manning WJ, Pohost GM, et al. (2004) Clinical indications for cardiovascular magnetic resonance (CMR): Consensus panel report. European Heart Journal 25:1940-1965.

4. Bull S, White SK, Piechnik SK, Flett AS, Ferreira VM, et al. (2013) Human non-contrast $\mathrm{T} 1$ values and correlation with histology in diffuse fibrosis. Heart 99: $932-937$

5. Moravsky G, Ofek E, Rakowski H, Butany J, Williams L, et al. (2013) Myocardia fibrosis in hypertrophic cardiomyopathy: accurate reflection of histopathological findings by CMR. J Am Coll Cardiol Img 6: 587-596.

6. Papavassiliu T, Schnabel P, Schröder M, Borggrefe M (2005) CMR scarring in a patient with hypertrophic cardiomyopathy correlates well with histological findings of fibrosis. Eur Heart J 26: 2395.
7. Barone-Rochette G, Pierard S, De Meester de Ravenstein C, Seldrum S Melchior J, et al. (2014) Prognostic significance of LGE by CMR in aortic stenosis patients undergoing valve replacement. J Am Coll Cardiol 64:144-154.

8. Azarisman SM, Teo KS, Worthley MI, Worthley SG (2014) Role of cardiovascular magnetic resonance in assessment of acute coronary syndrome. World $\mathrm{J}$ Cardiol 6: 405-414

9. Bhattacharyya S, Babu-Naravan SV, Chahal NS, Senior R, Li W (2013) Characterization of left ventricular myocardial fibrosis in adult congenital hear disease by contrast and deformation echocardiography validated by CMR. Int. J. Cardiol 166: 16-18.

10. Luu JM, Filipchuk NG, Friedrich MG (2013) Indications, safety and image quality of cardiovascular magnetic resonance: experience in $>5,000$ North American patients. Int J Cardiol 168: 3807-3811.

11. Kim YJ, Kim RJ (2011) The role of cardiac MR in new-onset heart failure. Curr Cardiol Rep 13: 185-193

12. Lepor NE, Pohost G, Gallik D (2014) Nonischemic dilated cardiomyopathy. Rev Cardiovasc Med 15: 73.

13. Gulati A, Jabbour A, Ismail TF, Guha K, Khwaja J, et al. (2013) Association of fibrosis with mortality and sudden cardiac death in patients with nonischemic dilated cardiomyopathy. JAMA 309: 896-908.

14. Hunt SA, Abraham WT, Chin MH, Feldman AM, Francis GS, et al. (2009) 2009 Focused update incorporated into the ACC/AHA 2005 Guidelines for the Diagnosis and Management of Heart Failure in Adults A Report of the American College of Cardiology Foundation/American Heart Association Task Force on Practice Guidelines Developed in Collaboration With the International Society for Heart and Lung Transplantation. J Am Coll Cardiol 53: e1-1e90.

15. Aljaroudi WA, Flamm SD, Saliba W, Wilkoff BL, Kwon D (2013) Role of CMR imaging in risk stratification for sudden cardiac death. JACC Cardiovasc Imaging 6: 392-406.

16. Kirchhof P, Breithardt G, Eckardt L (2006) Primary prevention of sudden cardiac death. Heart 92: 1873-1878.

17. Andreu D Berruezo A, Ortiz-Perez JT, Silva E, Mont L, Borras R, et al. (2011) Interrogation of 3Delectroanatomic maps and magnetic resonance scar characterization into the navigation system to guide ventricular tachycardia ablation. Circ Arrhythm Electrophysiol 4:674-683.

18. Marra MP, DeLazzari M, Zorzi A, Migliore F, Zilio F, et al. (2014) Impact of the presence and amount of myocardial fibrosis by cardiac magnetic resonance on arrhythmic outcome and sudden cardiac death in nonischemic dilated cardiomyopathy. Heart 11:856-863.

19. Neilan TG, Coelho-Filho O, Danik S, Verdini DJ, Michifumi T, et al. (2012) The impact of myocardial scar by cardiac magnetic resonance in patients with nonlschmic dilated cardiomyopathy referred for an implantable cardioverter defibrillation for primary prevention of sudden cardiac death. JACC 59: 866. 